\title{
A 1-day visit in a severe asthma centre: effect on asthma control, quality of life and healthcare use
}

\author{
Akke-Nynke van der Meer ${ }^{1}$, Henk Pasma ${ }^{1}$, Wilma Kempenaar-Okkema ${ }^{1}$, \\ Jo-Anneke Pelinck ${ }^{2}$, Myrte Schutten $^{3}$, Huib Storm ${ }^{4}$ and Anneke ten Brinke ${ }^{1}$
}

Affiliations: ${ }^{1}$ Dept of Respiratory Medicine, Medical Centre Leeuwarden, Leeuwarden, The Netherlands. ${ }^{2}$ Dept of Medical Psychology, Medical Centre Leeuwarden, Leeuwarden, The Netherlands. ${ }^{3}$ Dept of Physiotherapy, Medical Centre Leeuwarden, Leeuwarden, The Netherlands. ${ }^{4}$ Dept of Clinical Chemistry, Medical Centre Leeuwarden, Leeuwarden, The Netherlands.

Correspondence: Akke-Nynke van der Meer, Henri Dunantweg 2, 8934 AD Leeuwarden, The Netherlands. E-mail: AkkeNynke.van.der.Meeraznb.nl

ABSTRACT Patients with uncontrolled asthma report ongoing symptoms, poor quality-of-life and extensive healthcare use (HCU) and might benefit from management by a specialised severe asthma team. It is unknown whether a one-time evaluation by asthma experts, without long-term supervision by a specialised team, provides favourable outcomes. We evaluated asthma control (Asthma Control Questionnaire; ACQ), quality-of-life (Asthma-related Quality of Life Questionnaire; AQLQ) and HCU before and 1 year after a 1-day visit programme in a severe asthma centre, including a multidisciplinary assessment resulting in a personalised management plan to be implemented by patients own pulmonologists.

40 uncontrolled asthma patients completed questionnaires (ACQ, AQLQ, HCU) at baseline, and 6 and 12 months follow-up.

ACQ improved from 2.6 (interquartile range 1.7-3.2) to $1.8(1.2-3.2)(\mathrm{p}=0.003)$ and AQLQ from 4.8 $(4.0-5.2)$ to $5.3(4.4-6.0)(\mathrm{p}<0.001)$. We found a reduction in patients with $\geqslant 2$ exacerbations $(95 \%$ versus $17 \% ; \mathrm{p}<0.001), \geqslant 1$ emergency room visit $(78 \%$ versus $37 \% ; \mathrm{p}<0.001)$ and $\geqslant 1$ hospitalisation $(47 \%$ versus $10 \% ; \mathrm{p}=0.001)$.

Evaluation of uncontrolled asthma patients in a 1-day visit programme in a severe asthma centre resulted in significant improvements in asthma control, quality-of-life and healthcare use after 1 year. This 1-day visit approach seems beneficial for uncontrolled asthma patients and might reduce their dependence on expensive treatment modalities and long-term management in specialised centres.

@ERSpublications

1 day in a severe asthma centre is sufficient to improve asthma control, quality of life and healthcare use http://ow.ly/nvyN3002etI

Editorial comment in Eur Respir J 2016; 48: 611-613.

This article has supplementary material available from erj.ersjournals.com

Received: Jan 292016 | Accepted after revision: April 182016 | First published online: June 232016

Clinical trial: This study is registered at www.trialregister.nl with identifier number NTR5522.

Conflict of interest: None declared.

Copyright OERS 2016 


\section{Introduction}

The majority of asthma patients can be adequately treated with inhaled corticosteroids (ICS) and bronchodilators. However, a significant subset of patients remains difficult to treat [1]. These patients report ongoing asthma symptoms, poor quality of life and extensive healthcare use (HCU) despite maximal treatment. This subgroup of patients is responsible for high direct and indirect healthcare costs and poses a major healthcare problem [2].

Poor control in these patients might be due to several factors, including incorrect diagnosis, poor adherence, undertreated asthma triggers and co-morbidities and psychosocial problems [3]. Only patients who, after an extensive assessment addressing these issues, still need high-intensity inhaled treatment or systemic corticosteroids to prevent their asthma from becoming uncontrolled or who remain uncontrolled despite this therapy should be labelled as "severe refractory" asthma patients, and are candidate for novel therapeutic approaches [3,4]. Therefore, in patients presenting with chronic severe asthma symptoms, a systematic approach, preferably multidisciplinary [5], is recommended by all international severe asthma guidelines [6-8]

Previous studies have shown that a standardised evaluation protocol helped to identify [9] and treat comorbidities and triggering factors in patients with uncontrolled asthma and that, following such a systematic assessment, more than $50 \%$ were no longer difficult to treat $[10,11]$. Recently, prospective data from a UK registry showed that management of patients with difficult asthma at dedicated severe asthma centres resulted in improvement in quality of life (QoL) and HCU [12]. So far, it is not known whether it is possible to achieve favourable results with a single extensive assessment in a specialised severe asthma centre and subsequent referral of patients to their own general pulmonologists.

In the present study, we evaluated asthma control, QoL and HCU in patients with uncontrolled asthma before and 1 year after a 1-day visit programme in a specialised severe asthma centre, which included a systematic multidisciplinary assessment resulting in a personalised management plan to be implemented by patients' own pulmonologists. In addition, we analysed whether specific characteristics could predict a better outcome.

\section{Methods}

In 2013, in our specialised severe asthma centre, a 1-day visit programme was initiated for the evaluation of patients with uncontrolled asthma by a multidisciplinary team, including pulmonologists, physiotherapists, clinical psychologist and specialised asthma nurses. Patients were systematically evaluated with particular attention to the confirmation of asthma diagnosis and the presence of contributing factors and comorbidities. Based on clinical and inflammatory parameters, an initial determination of asthma phenotype was made. Findings were discussed in the multidisciplinary team and a personalised management plan aiming to improve asthma outcomes was provided to the patient and referring pulmonologist. All patients were referred back to their own pulmonologist, sometimes after an optimisation period of up to 6 months. Only the small subset of patients who were eligible for treatment which was not available in their own hospital remained for follow up in our centre. Detailed information on the 1-day-visit programme as well as our report back to the referring pulmonologist (assessment and stepwise management plan) is described in the online supplementary material.

In this prospective observational cohort study, we included adult non-smoking patients with uncontrolled asthma referred by pulmonologists from several hospitals in the Netherlands between June 2013 and June 2014. Six and 12 months after the assessment patients were asked to complete questionnaires on asthma control, QoL, prednisolone use and HCU. The study was approved by the hospital medical ethics committee, and all patients gave their written informed consent. The cohort was registered in The Netherlands trial register: NTR5522.

All patients underwent an extensive clinical, functional and laboratory assessment [3]. Data on demographics, medical history, smoking history, body mass index, comorbidities, psychological functioning and potential contributing factors, as well as medication use (adherence and inhalation technique) were collected. Peripheral blood cell counts were measured and expressed as absolute numbers. Atopic status was assessed by total and specific IgE to a panel of common aeroallergens. Lung function testing included spirometry before and after $400 \mu \mathrm{g}$ inhaled salbutamol [13]. High-resolution computed tomography of the thorax, computed tomography of the sinuses and ear, nose and throat evaluation data from referring pulmonologist were used in the assessment and whenever indicated performed (again). 6-min walking distance (6MWD) $[14,15]$ was assessed according to American Thoracic Society criteria [16]. Airway inflammation was assessed by the level of exhaled nitric oxide (FeNO) [17] and cell differentials in induced sputum [18].

Patients completed the Asthma Quality of Life Questionnaire (AQLQ) [19], the Asthma Control Questionnaire (ACQ) [20] and a questionnaire on HCU [21] at baseline as well as at 6 and 12 months afterwards. 
Patients were considered adherent if the Medication Adherence Report Scale (MARS) score was $\geqslant 4.0$ [22] and ICS prescription filling was $\geqslant 80 \%$ [23]. Prescription refill rates were calculated from prescription records for a 12-month time period. Exacerbations were defined as episodes with worsening of asthma symptoms, requiring prednisolone bursts or doubling oral corticosteroids (OCS) maintenance dose. Patients were phenotypically divided into non-eosinophilic, early onset atopic or late-onset eosinophilic subtypes. We labelled patients as non-eosinophilic if they had blood eosinophils $<0.3 \times 10^{9} \mathrm{cells} \cdot \mathrm{L}^{-1}$ and FeNO $<25 \mathrm{ppb}$ and, if available, sputum eosinophils $<3 \%$ both at baseline assessment as well as in all measurements in the previous year. If they had blood eosinophils $\geqslant 0.3 \times 10^{9}$ cells. $\mathrm{L}^{-1}$ or $\mathrm{FeNO} \geqslant 50 \mathrm{ppb}$ or sputum eosinophils $\geqslant 3 \%$ they were considered eosinophilic subtypes [24]. Early onset was defined as start of asthma at age $<18$ years and late onset at $\geqslant 18$ years. Positive atopic status was defined as a score of $>0.35 \mathrm{kU} \cdot \mathrm{L}^{-1}$ for at least one of the specific IgE tested.

\section{Statistical analysis}

Baseline measurements were compared with follow-up measurements using Wilcoxon matched pairs testing or Chi-squared analyses, whenever appropriate. Spearman rank correlation coefficients were used to analyse the relationship between outcome variables and baseline variables. All analyses were performed using SPSS software, version 20 (IBM, Armonk, NY, USA).

\section{Results}

In the first year of this 1-day visit programme, 47 patients with uncontrolled asthma completed the systematic assessment in which $51 \%$ classified as severe asthma and $40 \%$ as difficult-to-treat asthma [4]. In $9 \%$, the diagnosis of asthma could not be confirmed. 40 (85\%) patients had 6 and 12 months' follow up data available and were eligible for entry in this study. Based on the previously described phenotype criteria, $35 \%$ of these patients were considered as early onset atopic asthma, $45 \%$ as late-onset eosinophilic asthma, $15 \%$ as non-eosinophilic asthma and 5\% could not be classified. 15 patients were considered eligible for omalizumab treatment, 10 as first step therapy, five as second step to start when still uncontrolled after optimisation of contributing factors. Three of these first 10 patients already had been treated with omalizumab by their own pulmonologist, but had discontinued it due to adverse events or lack of efficacy. After the assessment, $83 \%$ of the patients returned to their own pulmonologist provided with a personalised management plan and only seven patients remained for follow up in our centre (five anti-interleukin 5 trial, two anti-immunoglobulin $\mathrm{E}$ treatment).

\section{Baseline characteristics}

Patients were aged between 22 and 72 years and showed a female predominance with $52 \%$ of them being non-atopic and 63\% reporting an adult onset of their asthma (table 1). Patients used high doses of inhaled steroids (ICS) and $28 \%$ of the patients were on daily OCS. Prescription filling analysis showed that $58.6 \%$ of the patients were adherent to their high-dose ICS with a prescription filling rate of $\geqslant 80 \%$. An additional diagnosis potentially contributing to poor asthma control was found in the majority of patients, with chronic rhinosinusitis and dysfunctional breathing being the most prevalent. Adequate sputum samples were obtained in $58 \%$ of the patients, of which $74 \%$ showed elevated sputum eosinophils $(\geqslant 3 \%)$ despite high-dose treatment.

\section{Effects on asthma control, QoL, OCS dose and HCU}

Asthma control as assessed by ACQ score improved from 2.6 (interquartile range 1.7-3.2) at baseline to $1.9(1.0-2.9)$ at 6 months and $1.8(1.2-3.2)$ at 1 year. $(\mathrm{p}=0.003)$ (figure 1$) .53 \%$ of patients had a clinical relevant improvement of ACQ of $>0.5$ point at 1 year. In addition, the Juniper AQLQ total score improved from $4.8(4.0-5.2)$ at baseline to $5.4(4.8-5.9)$ at 6 months and $5.3(4.4-6.0)$ at 1 year. $(\mathrm{p}<0.001)$ (figure 2).

Though there were obvious changes in individual prednisolone dose (figure 3 ), for the total group, we found no differences in the dose taken at follow up compared with baseline $(0 \mathrm{mg}(0-5)$ versus $0 \mathrm{mg}(0-5)$; $\mathrm{p}=0.7)$, or in the proportion of patients dependent on daily OCS ( $28 \%$ versus $35 \%$; $\mathrm{p}=0.4)$. At 1 year followup, $15 \%$ of patients were treated with omalizumab whereas $13 \%$ were participating in an anti-IL5 trial.

With respect to $\mathrm{HCU}$, at 1 year follow up, the number of asthma-related visits and hospital admissions was significantly reduced (table 2). There was a significant decrease in patients who reported frequent $(\geqslant 2$ ) exacerbations (95\% versus $17 \% ; \mathrm{p}<0.001)$, $\geqslant 1$ emergency room visit $(78 \%$ versus $37 \% ; \mathrm{p}<0.001)$, or $\geqslant 1$ hospital admission in the previous year $(47 \%$ versus $10 \% ; \mathrm{p}=0.001)$ compared with baseline. These 40 patients together accounted for a total number of 197 asthma exacerbations, 165 emergency room visits and 53 hospital admissions in the year preceding the 1-day visit, which had reduced to 39, 21 and 4, respectively in the year following the assessment. 
TABLE 1 Baseline characteristics

Total group

\begin{tabular}{|c|c|}
\hline Patients n & 40 \\
\hline Male sex & 14 (35) \\
\hline Age years & $51 \pm 13$ \\
\hline BMI $\mathrm{kg} \cdot \mathrm{m}^{-2}$ & $29 \pm 7$ \\
\hline Smoking history pack-years & $1.0(0-9.3)$ \\
\hline OCS dependent & $11(28)$ \\
\hline Fluticasone equivalent ICS dose $\mu \mathrm{g}$ & $750(500-1000)$ \\
\hline Atopic status & $19(48)$ \\
\hline Adult-onset asthma & $25(63)$ \\
\hline ICS adherence \% & $82(38-104)$ \\
\hline \multicolumn{2}{|l|}{ Inhaler technique \% } \\
\hline Poor & 5 \\
\hline Moderate & 27 \\
\hline \multicolumn{2}{|l|}{ Self-management \% } \\
\hline Poor & 5 \\
\hline Moderate & 38 \\
\hline \multicolumn{2}{|l|}{ Potentially contributing diagnoses } \\
\hline Rhinosinusitis & $24(60)$ \\
\hline Gastro-oesophageal reflux & $13(33)$ \\
\hline OSAS & $9(23)$ \\
\hline Obesity & $12(30)$ \\
\hline Dysfunctional breathing & $19(48)$ \\
\hline Psychological dysfunction & $12(30)$ \\
\hline Post-bronchodilator FEV $1 \%$ pred & $80 \pm 20$ \\
\hline 6MWD m & $488 \pm 108$ \\
\hline 6MWD \% pred & $84 \pm 19$ \\
\hline FeNO ppb & $38(17-68)$ \\
\hline Blood eosinophils $\times 10^{9}$ cells $\cdot \mathrm{L}^{-1}$ & $0.2(0.1-0.6)$ \\
\hline Blood neutrophils $\times 10^{9}$ cells $\cdot \mathrm{L}^{-1}$ & $5.7(3.9-6.8)$ \\
\hline Total IgE kU.L & $129(46-395)$ \\
\hline Sputum eosinophils \% & $16(0.8-32)$ \\
\hline Sputum neutrophils \% & $45(23-64)$ \\
\hline
\end{tabular}

Data are presented as $\mathrm{n}(\%)$, mean \pm SD or median (interquartile range), unless otherwise stated. BMI: body mass index; OCS: oral corticosteroids; ICS: inhaled corticosteroids; OSAS: obstructive sleep apnoea syndrome; FEV1: forced expiratory volume in $1 \mathrm{~s} ; \%$ pred: percentage of predicted value; 6MWD: 6 min walking distance; FeNO: exhaled fraction of nitric oxide; ppb: parts per billion; Ig: immunoglobulin.

\section{Predictors of asthma outcomes}

Asthma outcomes (improvement in ACQ or AQLQ, reduction in numbers of exacerbations, emergency room visits or hospital admissions) were not dependent on sex, smoking history or whether patients were referred back to their own pulmonologist or not. Patients labelled as early onset atopic asthma, late-onset eosinophilic asthma and non-eosinophilic asthma all showed comparable beneficial effects after 1 year. A larger improvement in ACQ was seen in patients with higher 6MWD ( $r=-0.40, p=0.01)$, lower body mass

FIGURE 1 Asthma Control Questionnaire (ACQ) scores at baseline assessment (0 months) and 6 and 12 months follow-up in 40 patients with uncontrolled asthma. Data are presented as median (interquartile range). $\Delta 0.5$ : difference of $\geqslant 0.5$ indicating a clinically significant improvement in asthma control.

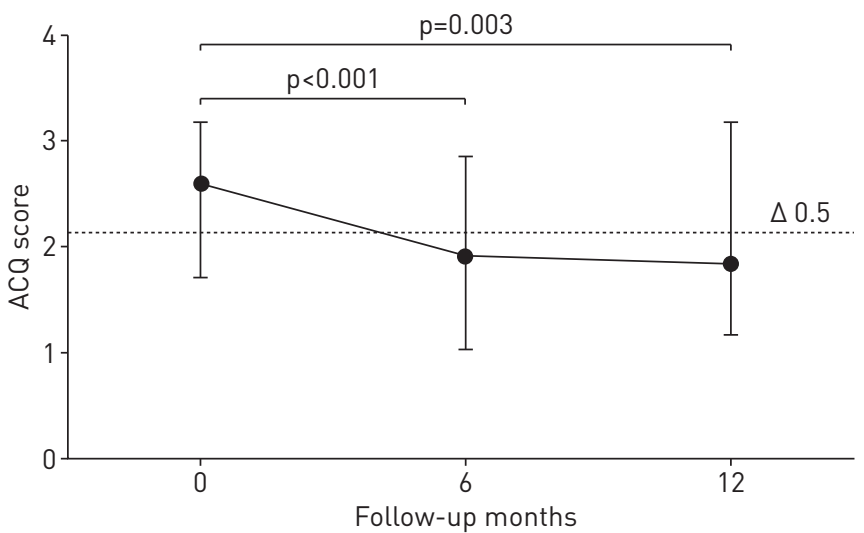




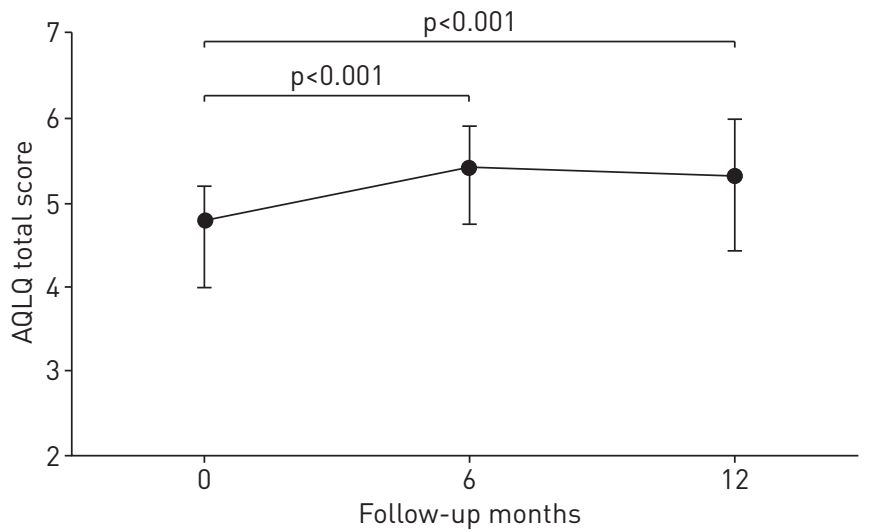

FIGURE 2 Asthma-related Quality of Life Questionnaire (AQLQ) scores at baseline assessment (0 months) and 6 and 12 months follow-up in 40 patients with uncontrolled asthma. Data are presented as median (interquartile range).

index $(\mathrm{r}=0.34, \mathrm{p}=0.03)$ and higher levels of sputum eosinophils $(\mathrm{r}=-0.41, \mathrm{p}=0.05)$ at baseline. In addition, the reduction in exacerbations was related to higher baseline levels of FeNO $(\mathrm{r}=-0.34, \mathrm{p}=0.03)$ and eosinophils in blood $(\mathrm{r}=-0.32, \mathrm{p}=0.04)$ as well as in sputum $(\mathrm{r}=-0.43, \mathrm{p}=0.04)$.

\section{Discussion}

In the present study, we show that patients with uncontrolled asthma benefit from a single extensive assessment in a specialised severe asthma centre, with a significant and clinically relevant improvement in asthma control, quality of life and HCU after 1 year. In the current 1-day visit programme, patients were systematically evaluated by a multidisciplinary team, referred back to their own pulmonologists and provided with a personalised management plan. Compared with the year preceding the assessment, the number of exacerbations, emergency room visits and hospital admissions was reduced by $54 \%, 57 \%$ and $43 \%$, respectively in the 12 months' follow up. Asthma outcomes were not dependent on sex, smoking history or phenotype. The greatest improvements in asthma control and exacerbation frequency were seen in the patients with higher baseline sputum eosinophils. These results suggest that a single short-term extensive characterisation in a specialised severe asthma centre is beneficial and might be cost effective for a large group of patients with uncontrolled asthma.

In our study, we evaluated the effect of characterising patients with uncontrolled asthma by a dedicated severe asthma team and observed rather impressive improvements in asthma outcomes that persisted long after the patients were referred back to their own pulmonologists. A recent UK registry study [12] showed that management of severe asthma patients in specialised severe asthma centres was associated with improvement in asthma control, QoL and HCU, but data about how long patients visited these clinics and were managed by a specialised team were not mentioned. The present study largely confirmed their results with even more favourable effects on exacerbation and admission rates; although we found no change in daily dose of prednisolone. Our results further highlight that all phenotypes appear to benefit, with the most positive effects for patients with eosinophilic airway inflammation at baseline. The presented standardised 1-day visit approach adds a new component that hopefully contributes to a wider application of the comprehensive characterisation of patients with uncontrolled asthma by a specialised team.

The strength of this study lies in the extensive and validated description of all relevant patient characteristics, including questionnaires, allergy testing, spirometry, induced sputum and blood cell counts,

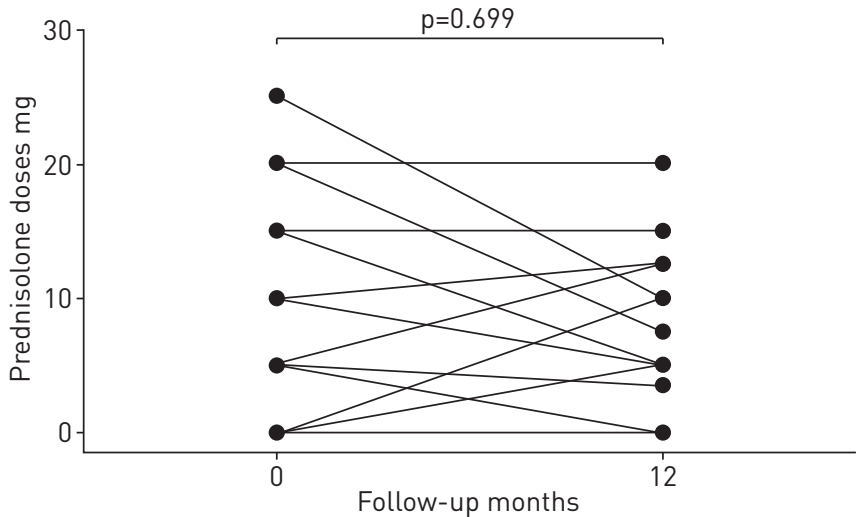

FIGURE 3 Changes in prednisolone dose at baseline assessment and 12 months follow-up in 40 patients with uncontrolled asthma. 


\begin{tabular}{|c|c|c|c|}
\hline During preceding year & Baseline & 1 year & p-value \\
\hline Asthma related GP visits & $3(2-7)$ & $1(0-3)$ & 0.001 \\
\hline Pulmonologist visits & $4(3-8)$ & $3(2-4)$ & 0.005 \\
\hline Exacerbations & $4.5(2-7)$ & $1(0-3)$ & $<0.001$ \\
\hline Emergency room visits & $3(1-6)$ & $0.5(0-2)$ & $<0.001$ \\
\hline Hospital admissions & $0(0-2)$ & $0(0-0)$ & $<0.001$ \\
\hline
\end{tabular}

Data are presented as median (interquartile range). GP: general practitioner.

psychological evaluation and 6MWD. This comprehensive systematic characterisation by a dedicated team using pre-established criteria and definitions reduces the risk of bias due to non-standardised approaches and diverse interpretations by different healthcare professionals.

We acknowledge there are several limitations in our study. Firstly, the classification of patients as difficultto-treat or severe asthma. In our programme, we labelled $51 \%$ of the patients as severe asthma without having a 3-month follow-up period in our specialised centre, as has been recommended in guidelines [4]. This period is mainly recommended to evaluate the patients regarding appropriate diagnosis and/or treatment of confounders. In our setting, all patients were followed for several years by a pulmonologist who had already performed this evaluation to a greater or lesser extent. After our 1-day-visit programme, only the patients with confirmed asthma diagnosis, uncontrolled disease despite high doses of medication, good adherence and inhalation technique, and optimised comorbid factors/confounders were considered as severe asthma. All others were labeled as difficult asthma, for the time being, and treated for the observed potentially contributing factors. After addressing these factors we still expect some of these patients to come out as truly severe asthma patients. Secondly, the data on HCU are based on self-report, and may be influenced by recall bias. Recall of HCU data in respiratory patients is fairly reliable for hospitalisations and visits to pulmonologists [25], whereas for emergency room visits, a bias towards under-reporting has been suggested, particular at higher numbers of visits [26]. Although depending on the objective of the analysis, the chosen recall period may be more or less optimal [27], we expect a possible recall bias mainly to underestimate the dimension of the problem and not to explain the large differences in HCU we observed in the two periods. Thirdly, there are not currently widely accepted definitions of specific asthma phenotypes. In the present study, we found no differences in asthma outcomes between the three phenotypes we defined, but we cannot rule out that adjustment of the criteria for distinct phenotypes could lead to different results. Finally, the absence of a control group is obvious. Improvements in quality of life could be attributed to the fact that patients received more attention and additional tests from different healthcare providers, but it is doubtful whether this may be responsible for the improvements after 1 year. Though we strongly believe that the given insight into their thus far uncontrollable disease contributes to patients' well-being and might have improved their adherence to therapy, we don't expect a placebo effect to explain the beneficial effects measured long after the patients were discharged from our centre.

What other reasons might explain the significant improvements in asthma control, QoL and HCU? The recommendations given in the personalised management plans encompassed various interventions, varying from optimising triggering and comorbid factors [9, 10], improving inhalation technique and adherence [28], increasing physical or psychological functioning to changes in asthma medication. Following our assessment, seven patients started omalizumab treatment and five patients participated in a placebo-controlled trial with mepolizumab, both drugs that are associated with reduction in exacerbation frequency and improvement in QoL [29, 30]. For patients who were not eligible for these biologicals, beneficial effects might further be attributed to the phenotype-specific approach, in which the presence or absence of eosinophilic inflammation played a crucial role [31]. Without evidence for eosinophilic inflammation at time of assessment or in the preceding years it was strongly advocated to taper the, in some cases very high, doses of oral and inhaled corticosteroids. Alternatively, patients with eosinophilic inflammation despite extensive treatment were encouraged to start or increase prednisolone as maintenance therapy, pending the availability of new biologicals. This approach of "giving prednisolone to the right patients" as reflected in figure 3, might have contributed to the better outcomes even though the mean prednisolone dose did not change. The finding that exacerbation frequency was most reduced in patients with more active eosinophilic inflammation further supports our phenotype-specific eosinophil-driven treatment.

The present findings are clinically relevant for the management of patients with uncontrolled asthma. Anticipating several novel molecular therapies we face the challenge to limit the costs of uncontrolled 
asthma treatment by making these expensive drugs available only for patients with truly severe asthma. We show that a comprehensive characterisation of patients with uncontrolled asthma by a specialised team is very successful in improving the condition of a majority of patients, thereby reducing the need for new expensive therapies. More important, our study showed that these favourable results were achieved by a single short-term assessment in a severe asthma centre, even though the implementation of the recommended personalised management plan was not supervised by severe asthma specialists. Assessments using a 1-day visit programme may facilitate the evaluation of uncontrolled patients by a specialised team, both by reduction of travel distances for patients, as well as by limiting time investment of severe asthma specialists. The costs of such a 1-day visit programme seem justified in view of the anticipated reduced use of expensive asthma drugs and the observed benefits in terms of healthcare use, asthma control and quality of life.

In conclusion, in the present prospective study patients with uncontrolled asthma who were systematically evaluated by a 1-day visit programme in a specialised severe asthma centre showed a significant and clinically relevant improvement in asthma control, QoL and HCU lasting up to 12 months. These results suggest that a single visit with extensive characterisation in a dedicated severe asthma centre is beneficial and sufficient for a large group of patients with uncontrolled asthma, thereby reducing the number of patients that depend on expensive treatment modalities and continuous management in a specialised centre.

\section{Acknowledgements}

Author contributions: A-N. van der Meer, H. Pasma, W. Kempenaar, J-A. Pelinck, M. Schutten and A. ten Brinke collected the data, A-N. van der Meer and A. ten Brinke analysed the data and wrote the paper. A. ten Brinke was responsible for the design of the study, and $\mathrm{H}$. Pasma and $\mathrm{H}$. Storm revised the article critically. All approved this version to be published.

\section{References}

1 Bateman ED, Boushey HA, Bousquet J, et al. Can guideline-defined asthma control be achieved? The Gaining Optimal Asthma ControL study. Am J Respir Crit Care Med 2004; 170: 836-844.

2 Braman SS. The global burden of asthma. Chest 2006; 130: Suppl, 4-12.

3 Bel EH, Sousa A, Fleming L, et al. Diagnosis and definition of severe refractory asthma: an international consensus statement from the Innovative Medicine Initiative (IMI). Thorax 2011; 66: 910-917.

4 Bousquet J, Khaltaev N, Cruz A, et al. International European Respiratory Society/American Thoracic Society guidelines on severe asthma. Eur Respir J 2014; 44: 1377-1378.

McDonald VM, Vertigan AE, Gibson PG. How to set up a severe asthma service. Respirology 2011; 16: 900-911.

Thomson CC, Welsh CH, Carno MA, et al. Severe asthma. Ann Am Thorac Soc 2014; 11: 996-997.

British Thoracic Society, Scottish Intercollegiate Guidelines Network. British guideline on the management of asthma. Thorax 2014; 69: Suppl 1, 1-92.

8 Global Initiative for Asthma (GINA) 2015. Global strategy for asthma management and prevention. Available from www.ginasthma.org Date last accessed: 2015. Date last updated: 2016.

9 ten Brinke A, Sterk PJ, Masclee AAM, et al. Risk factors of frequent exacerbations in difficult-to-treat asthma. Eur Respir J 2005; 26: 812-818.

10 Irwin RS, Curley FJ, French CL. Difficult-to-control asthma. Contributing factors and outcome of a systematic management protocol. Chest 1993; 103: 1662-1669.

11 Heaney LG, Conway E, Kelly C, et al. Predictors of therapy resistant asthma: outcome of a systematic evaluation protocol. Thorax 2003; 58: 561-566.

12 Gibeon D, Heaney LG, Brightling CE, et al. Dedicated severe asthma services improve health-care use and quality of life. Chest 2015; 148: 870-876.

13 Quanjer PH, Tammeling GJ, Cotes JE, et al. [Lung volumes and forced ventilatory flows. Work Group on Standardization of Respiratory Function Tests. European Community for Coal and Steel. Official position of the European Respiratory Society]. Rev Mal Respir 1994; 11: Suppl 3, 5-40.

14 Butland RJ, Pang J, Gross ER, et al. Two-, six-, and 12-minute walking tests in respiratory disease. $\mathrm{Br}$ Med J 1982; 284: $1607-1608$

15 O'Keeffe ST, Lye M, Donnellan C, et al. Reproducibility and responsiveness of quality of life assessment and six minute walk test in elderly heart failure patients. Heart 1998; 80: 377-382.

16 ATS Committee on Proficiency Standards for Clinical Pulmonary Function Laboratories. ATS statement: guidelines for the six-minute walk test. Am J Respir Crit Care Med 2002; 166: 111-117.

17 American Thoracic Society, European Respiratory Society. ATS/ERS recommendations for standardized procedures for the online and offline measurement of exhaled lower respiratory nitric oxide and nasal nitric oxide, 2005. Am J Respir Crit Care Med. 2005; 171: 912-930.

18 ten Brinke A, de Lange C, Zwinderman AH, et al. Sputum induction in severe asthma by a standardized protocol: predictors of excessive bronchoconstriction. Am J Respir Crit Care Med 2001; 164: 749-753.

19 Juniper EF, Svensson K, Mörk AC, et al. Modification of the asthma quality of life questionnaire (standardised) for patients 12 years and older. Health Qual Life Outcomes 2005; 3: 58.

20 Juniper EF, O'Byrne PM, Guyatt GH, et al. Development and validation of a questionnaire to measure asthma control. Eur Respir J 1999; 14: 902-907.

21 ten Brinke A, Ouwerkerk ME, Zwinderman AH, et al. Psychopathology in patients with severe asthma is associated with increased health care utilization. Am J Respir Crit Care Med 2001; 163: 1093-1096.

22 Cohen JL, Mann DM, Wisnivesky JP, et al. Assessing the validity of self-reported medication adherence among inner-city asthmatic adults: the Medication Adherence Report Scale for Asthma. Ann Allergy Asthma Immunol 2009; 103: 325-331 
23 McNicholl DM, Stevenson M, McGarvey LP, et al. The utility of fractional exhaled nitric oxide suppression in the identification of nonadherence in difficult asthma. Am J Respir Crit Care Med 2012; 186: 1102-1108.

24 Pavord ID, Korn S, Howarth P, et al. Mepolizumab for severe eosinophilic asthma (DREAM): a multicentre, double-blind, placebo-controlled trial. Lancet 2012; 380: 651-659.

25 Ungar W. Bias--it's everywhere! A commentary on the impact of bias and the assessment of agreement in the measurement of medication use in epidemiology research. Pharmacoepidemiol Drug Saf 1998; 7: 425-427.

26 Roberts RO, Bergstralh EJ, Schmidt L, et al. Comparison of self-reported and medical record health care utilization measures. J Clin Epidemiol 1996; 49: 989-995.

27 Kjellsson G, Clarke P, Gerdtham UG. Forgetting to remember or remembering to forget: a study of the recall period length in health care survey questions. J Health Econ 2014; 35: 34-46.

28 Kim C, Feldman HI, Joffe M, et al. Influences of earlier adherence and symptoms on current symptoms: a marginal structural models analysis. J Allergy Clin Immunol 2005; 115: 810-814.

29 Finn A, Gross G, van Bavel J, et al. Omalizumab improves asthma-related quality of life in patients with severe allergic asthma. J Allergy Clin Immunol 2003; 111: 278-284.

30 Bel EH, Wenzel SE, Thompson PJ, et al. Oral glucocorticoid-sparing effect of mepolizumab in eosinophilic asthma. N Engl J Med 2014; 371: 1189-1197.

31 Green RH, Brightling CE, McKenna S, et al. Asthma exacerbations and sputum eosinophil counts: a randomised controlled trial. Lancet 2002; 360: 1715-1721. 